\title{
Influence de la forme de présentation et de la finesse de mouture de l'aliment sur les performances zootechniques et la fonction cæcale chez le lapin en croissance
}

\author{
M. CANDAU, A. AUVERGNE, F. COMES, M. BOUILliER-OUdOT \\ avec la collaboration technique de G. LATIL \\ Ecole Nationale Supérieure Agronomique \\ Service de Zootechnie et des Productions Animales \\ 145, avenue de Muret, 31076 Toulouse Cedex, France
}

\begin{abstract}
Résumé
Les effets de la forme de présentation (granulés vs farine) et de la finesse de mouture des aliments avant granulation (4 granulométries différentes), sont étudiés dans deux essais en enregistrant les performances zootechniques et les caractéristiques du cæcum à l'abattage.

La distribution d'aliments sous forme de farine conduit à une sous-consommation sans troubles respiratoires.

L'hétérogénéité des aliments non granulés favorise le tri des matières premières et diminue l'indice de consommation. La granulation d'un aliment à partir d'une mouture fine permet les meilleures performances. A l'abattage, les contenus cæcaux des animaux ayant reçu cet aliment ont un $\mathrm{pH}$ plus acide et des concentrations en acides gras volatils plus élevées.

A l'inverse, les performances les plus faibles, associées à un $\mathrm{pH}$ élevé du contenu cæcal et une déviation fermentaire ( $\%$ de $\mathrm{C}_{4}$ diminué, $\%$ de $\mathrm{C}_{2}$ augmenté) sont observées avec des aliments présentés sous forme de farine grossière.
\end{abstract}

Mots clés : Alimentation, lapin, farine, granulométrie, cocum.

\section{Introduction}

Chez le porc, la fabrication de l'aliment à la ferme est possible en raison de l'adaptation comportementale et physiologique de cette espèce à différentes formes de présentation de l'aliment (Braude et Rowell, 1966 ; BaIRD, 1973 ; Aumaitre et al., 1977). Or, s'il a été montré que le lapin était susceptible d'ingérer un aliment présenté sous forme de farine (Lebas, 1973), l'économie du coût technologique de la granulation peut être annulée par des performances de croissance plus faibles et une augmentation des risques pathologiques, liée à un effet de la taille des particules constitutives de l'aliment (LAPlace et LeBAS, 1977). Ceci pose le problème, chez cet animal, de l'adéquation de la granulométrie de l'aliment à un équilibre digestif toujours précaire dont la rupture peut se manifester aussi bien par une diarrhée que par une constipation. 
Le présent travail a pour but, au travers de deux essais, d'analyser chez le lapin, ces relations en faisant varier la forme de présentation (farine ou granulés) de l'aliment et la finesse de mouture des matières premières (céréales, pois, luzerne).

\section{Matériel et méthodes}

\section{A. Animaux}

Les animaux utilisés sont des lapins en croissance, âgés de 28 à 71 jours, élevés en cages communautaires de 4 individus, sexes mélangés. La mise en lot est réalisée selon la méthode décrite par Morin et al. (1979). Le premier essai comporte 4 répétitions de 16 lapins, soit 64 animaux au total, le second 5 répétitions de 16 lapins, soit 80 animaux.

\section{B. Aliments utilisés}

Le but de cet essai étant de valoriser des matières premières produites sur l'exploitation, nous avons retenu une formule alimentaire à base de luzerne, de pois fourrager, d'orge et de maïs.

La luzerne est apportée sous forme de bouchons déshydratés ou de foin. La composition des aliments et les traitements technologiques des matières premières sont reportés dans le tableau 1.

Les différentes matières premières sont broyées isolément par un broyeur à marteaux (Electra Scté-47 Poudenas) et mélangés soit dans une bétonnière (essai 1) par unité de $40 \mathrm{~kg}$ environ, soit dans un mélangeur vertical (essai 2) par unité de $300 \mathrm{~kg}$ environ.

Le premier essai vise à évaluer les possibilités d'alimentation du lapin avec des mélanges concassés ou avec des mélanges de farines grossièrement moulues. Les céréales et le pois sont concassés au broyeur, tournant à $1500 \mathrm{t} / \mathrm{mn}$, et muni d'une grille de $6 \mathrm{~mm}$. Les mêmes matières premières sont également préparées par mouture à $6000 \mathrm{t} / \mathrm{mn}$ (broyeur muni d'une grille de $6 \mathrm{~mm}$ ). La luzerne déshydratée est présentée soit en bouchons éclatés par passage au broyeur $(1500 \mathrm{t} / \mathrm{mn})$ sans grille, soit en farine grossière (en équipant le broyeur d'une grille de $8 \mathrm{~mm}$ ). Quatre aliments sont ainsi définis pour estimer l'effet de la mouture et de l'hétérogénéité des mélanges présentés sous forme de farine.

Dans le second essai, est évaluée l'influence de la forme de présentation de l'aliment, granulé ou farine (aliment A vs aliment B), de la finesse de mouture (aliment $B$ vs aliment $C$ ), de la nature de la luzerne, foin ou farine déshydratée (aliment $C$ vs aliment D).

Pour ce faire, les matières premières des aliments $A$ et $B$ sont broyées à $6000 \mathrm{t} /$ mn (broyeur muni d'une grille de $3 \mathrm{~mm}$ ) et mélangées ; la moitié de ce mélange étant granulé (Station d'élevage du lapin INRA-Auzeville). Pour les aliments C et D, le broyeur est muni d'une grille de $6 \mathrm{~mm}$. 


\section{TABleAU 1}

Composition des aliments et traitements technologiques des matières premières.

Diet composition and technological treatments of raw matters.

\begin{tabular}{|c|c|c|c|c|c|c|c|c|}
\hline \multirow{2}{*}{$\frac{\text { Essai / Trial }}{\text { Régimes / Diets }}$} & \multicolumn{4}{|c|}{ I } & \multicolumn{4}{|c|}{ II } \\
\hline & $\mathbf{I}$ & II & III & IV & A & B & $\mathrm{C}$ & D \\
\hline \multicolumn{9}{|l|}{$\begin{array}{l}\text { Composition pondérale (p. 100) } \\
\text { Composition of diets }\end{array}$} \\
\hline $\begin{array}{l}\text { Luzerne déshydratée } \ldots \ldots \ldots \ldots \\
\text { Dehydrated lucerne }\end{array}$ & \multicolumn{4}{|c|}{30} & - & - & - & 40 \\
\hline $\begin{array}{l}\text { Foin de luzerne } \ldots \ldots \ldots \ldots \ldots \\
\text { Lucerne hay }\end{array}$ & \multicolumn{4}{|c|}{ - } & 40 & 40 & 40 & - \\
\hline $\begin{array}{l}\text { Pois fourrager } \\
\text { Peas }\end{array}$ & \multicolumn{4}{|c|}{33} & 29,3 & 29,3 & 29,3 & 29,3 \\
\hline$\underset{B \text { Barley }}{\text { Orge }} \ldots \ldots \ldots \ldots \ldots \ldots$ & \multicolumn{4}{|c|}{15} & 29,3 & 29,3 & 29,3 & 29,3 \\
\hline $\begin{array}{l}\text { Maïs } \ldots \ldots \ldots \ldots \ldots \ldots \ldots \\
\text { Maize }\end{array}$ & \multicolumn{4}{|c|}{15} & - & - & - & - \\
\hline $\begin{array}{l}\text { Complément minéral et vitaminé } \\
\text { Vitamin and Mineral mixture } \\
\text { (CMV) } \neq 1 \ldots \ldots \ldots \ldots \ldots \ldots\end{array}$ & \multicolumn{4}{|c|}{$\begin{array}{l}7 \\
-\end{array}$} & $\overline{1,4}$ & $\overline{1,4}$ & $\overline{1,4}$ & $\overline{1,4}$ \\
\hline \multicolumn{9}{|l|}{ Traitements / Treatments } \\
\hline $\begin{array}{l}\text { Mouture des grains } \ldots \ldots \ldots \ldots \\
\text { Grain particle size }\end{array}$ & \multicolumn{2}{|c|}{$\begin{array}{l}\text { concassé } \\
\text { crushed } \\
1500 \mathrm{t} / \mathrm{mn}\end{array}$} & \multicolumn{2}{|c|}{$\begin{array}{l}\text { broyé } 6 \mathrm{~mm} \\
\text { ground } \\
6000 \mathrm{t} / \mathrm{mn}\end{array}$} & \multicolumn{2}{|c|}{$\begin{array}{l}\text { broyé } 3 \mathrm{~mm} \\
\text { ground } 3 \mathrm{~mm}\end{array}$} & \multicolumn{2}{|c|}{$\begin{array}{l}\text { broyé } 6 \mathrm{~mm} \\
\text { ground } 3 \mathrm{~mm}\end{array}$} \\
\hline $\begin{array}{l}\text { de la luzerne } \ldots \ldots \ldots \\
\text { of lucerne }\end{array}$ & $\underset{(1)}{\text { Eclat }}$ & $\begin{array}{l}\text { Gros. } \\
(2)\end{array}$ & $\begin{array}{c}\text { Eclat } \\
(1)\end{array}$ & Gros. & 6000 & $t / m n$ & 6000 & $\mathrm{t} / \mathrm{mn}$ \\
\hline $\begin{array}{l}\text { Présentation } \\
\text { Physical form }\end{array}$ & $\begin{array}{r}\text { Méla } \\
\text { Unc }\end{array}$ & $\begin{array}{l}\text { iges no } \\
\text { ompress }\end{array}$ & $\begin{array}{l}\text { agglor } \\
\text { ed mixt }\end{array}$ & $\begin{array}{l}\text { nérés } \\
\text { ures }\end{array}$ & (3) & & $\begin{array}{l}\text { Farine: } \\
\text { Flours }\end{array}$ & \\
\hline
\end{tabular}

Eclat : bouchons de luzerne éclatés (broken pellets of lucerne).

Gros. : mouture grossière (coarse grinding)

CMV $\neq 1: 23$ p. 100 maïs, 23 p. 100 tourteau d'arachide, 11 p. $100 \mathrm{NaCl}, 35$ p. 100 phosphate monobicalcique, 1 p. 100 complément oligovitaminé, 1 p. 100 L-lysine, 1 p. 100 DL-méthionine.

$\mathrm{CMV} \neq 2: 83$ p. 100 phosphate monosodique, 3 p. 100 complément oligovitaminé, 13 p. 100 DL-méthionine. (1) Broken - (2) Coarse - (3) Granulés / Pellets

\section{Mesures effectuées}

Les aliments sont distribués trois fois par semaine, la consommation étant enregistrée le jour de la pesée hebdomadaire. L'analyse fourragère classique est effectuée selon les méthodes européennes (1971, 1972, 1973). La teneur en constituants pariétaux est déterminée par la méthode de Van Soest (attaque au détergent lauryl sulfate en milieu neutre, 1967). La répartition granulométrique des différents aliments est estimée sur des échantillons cumulés prélevés à chaque distribution. Les mesures de granulomé- 
trie sont obtenues par séparation des farines avant granulation sur des tamis à mailles carrées de $4,0,2,0,1,0,0,5,0,315,0,100 \mathrm{~mm}$ de côté, à l'aide d'une tamiseuse à mouvement tridimentionnel.

Les lapins sont abattus à 71 jours d'âge. On mesure alors le poids vif, le poids P.A.C. (prêt à cuire), le poids du cæcum plein, le poids du contenu cæcal frais. Le $\mathrm{pH}$ du contenu cæcal est apprécié in situ à l'aide d'une électrode à milieu pâteux (référence Ingold G 90408) dans les 5 minutes qui suivent la mort de l'animal. Deux échantillons de contenu cacal sont prélevés pour la détermination du taux de matière sèche (détermination par passage à l'étuve à $103^{\circ} \mathrm{C}$ et par pesée à poids constant) et de la concentration en acides gras volatils (par chromatographie en phase gazeuse sur un chromatographe Intersmat IGC $120 \mathrm{~B}$, phase stationnaire 10 p. 100 SP $1200 / 1$ p. 100 $\mathrm{H}_{3} \mathrm{PO}_{4}$, support chromosorb W.AW 82/100, étalon interne acide isobutyrique).

L'utilisation de farine ayant la réputation de provoquer des problèmes respiratoires chez le lapin, les poumons sont examinés systématiquement après éviscération et notés de 0 à 4 en fonction de la gravité des atteintes pulmonaires selon une grille inspirée de celle utilisée pour le porc (document ITP 1981) (tabl. 2).

Les résultats sont traités par la méthode d'analyse de variance à deux voies pour le premier essai et à une voie pour le second.

TABleau 2

Grille de classement sanitaire des poumons.

Lung health grading scale.

\begin{tabular}{c|l}
\hline \hline Note / Score & \multicolumn{1}{c}{ Critères de classement / Grading criteria } \\
\hline 0 & $\begin{array}{l}\text { Pas d'atteinte pulmonaire (congestion, pneumonie ou atelectasie) } \\
\text { No lung damage (congestion, pneumonia or atelectasia) }\end{array}$ \\
\hline 1 & $\begin{array}{l}\text { Quelques points circonscrits sur un seul lobe } \\
\text { Slight damage of one lobe }\end{array}$ \\
\hline 2 & $\begin{array}{l}\text { Quelques points circonscrits sur deux lobes ou plus } \\
\text { Slight damage of at least two lobes }\end{array}$ \\
\hline 3 & $\begin{array}{l}\text { Atteinte étendue sur plusieurs lobes intéressant moins d'1/4 des poumons } \\
\text { Large damage of some lobes, but less than 1/4 of the lungs }\end{array}$ \\
\hline 4 & $\begin{array}{l}\text { Atteinte importante intéressant plus d'1/4 des poumons } \\
\text { Large damage of more than 1/4 of the lungs }\end{array}$ \\
\hline
\end{tabular}

\section{Résultats}

\section{A. Composition des aliments expérimentaux}

Les aliments de l'essai 1 sont sensiblement izoazotés et isocellulosiques (tabl. 3). Dans l'essai 2, l'aliment $\mathrm{D}$ à base de luzerne déshydratée a une teneur en azote plus 
TABleau 3

Composition chimique des aliments expérimentaux ( $p .100$ de la matière sèche). Chemical composition of diets (p. 100 of dry matter).

\begin{tabular}{|c|c|c|c|c|c|c|c|c|}
\hline \multirow[b]{2}{*}{ Régimes / Diets } & \multicolumn{4}{|c|}{ Essai 1} & \multicolumn{4}{|c|}{ Essai 2} \\
\hline & $\mathbf{I}$ & II & III & IV & A & B & $\mathrm{C}$ & $\mathrm{D}$ \\
\hline $\begin{array}{l}\text { Matière sèche } \ldots \ldots \ldots \\
\text { Dry matter }\end{array}$ & 89,9 & 87,4 & 87,6 & 90,4 & 88,2 & 84,0 & 84,6 & 87,6 \\
\hline $\begin{array}{l}\text { Matières minérales } \\
\text { Ash }\end{array}$ & 7,7 & 7,5 & 7,9 & 7,6 & 7,1 & 7,3 & 8,5 & 6,5 \\
\hline $\begin{array}{l}\text { Matières azotées totales } \\
\text { Crude protein }\end{array}$ & 13,8 & 14,5 & 14,4 & 13,3 & 17,2 & 17,3 & 18,1 & 14,3 \\
\hline $\begin{array}{l}\text { Cellulose brute } \ldots \ldots \ldots \\
\text { Crude fibre }\end{array}$ & 12,3 & 13,2 & 12,3 & 12,8 & 12,4 & 12,8 & 12,7 & 13,7 \\
\hline Neutral detergent fiber. & 29,3 & 31,5 & 39,7 & 31,0 & 34,5 & 34,3 & 33,2 & 46,3 \\
\hline $\begin{array}{l}\text { Matières grasses } \ldots \ldots \cdots \\
\text { Fat }\end{array}$ & 2,0 & 2,1 & 1,6 & 2,3 & 2,0 & 1,7 & 1,7 & 1,4 \\
\hline
\end{tabular}

faible mais surtout un taux de glucides pariétaux plus élevé. Ceci est à relier à la qualité médiocre de cette luzerne comparée à celle du foin de luzerne "soleil »utilisé dans les aliments $\mathrm{A}, \mathrm{B}$ et $\mathrm{C}$.

L'analyse granulométrique des aliments (fig. 1) permet de constater que dans l'essai 1, les tailles de particules des aliments I et II (graines et grains concassés, luzerne en bouchons éclatés ou moulus grossièrement) sont voisins. Les pourcentages de particules grossières sont importants. Le fait de broyer (avec une grille de $6 \mathrm{~mm}$ ) les graines et grains des aliments III et IV déplace le spectre granulométrique vers des tailles de particules plus faibles. Dans l'essai 2, les aliments C et D se caractérisent par une granulométrie plus grossière que les aliments $\mathrm{A}$ et $\mathrm{B}, \mathrm{C}$ étant lui-même plus grossier que D.

\section{B. Performances zootechniques}

\section{Déroulement de l'essai}

Aucun effet significatif des traitements sur la mortalité n'est enregistré au cours de l'essai 1 (tabl. 4). Les animaux du lot II présentent cependant des atteintes pulmonaires significativement plus importantes. 


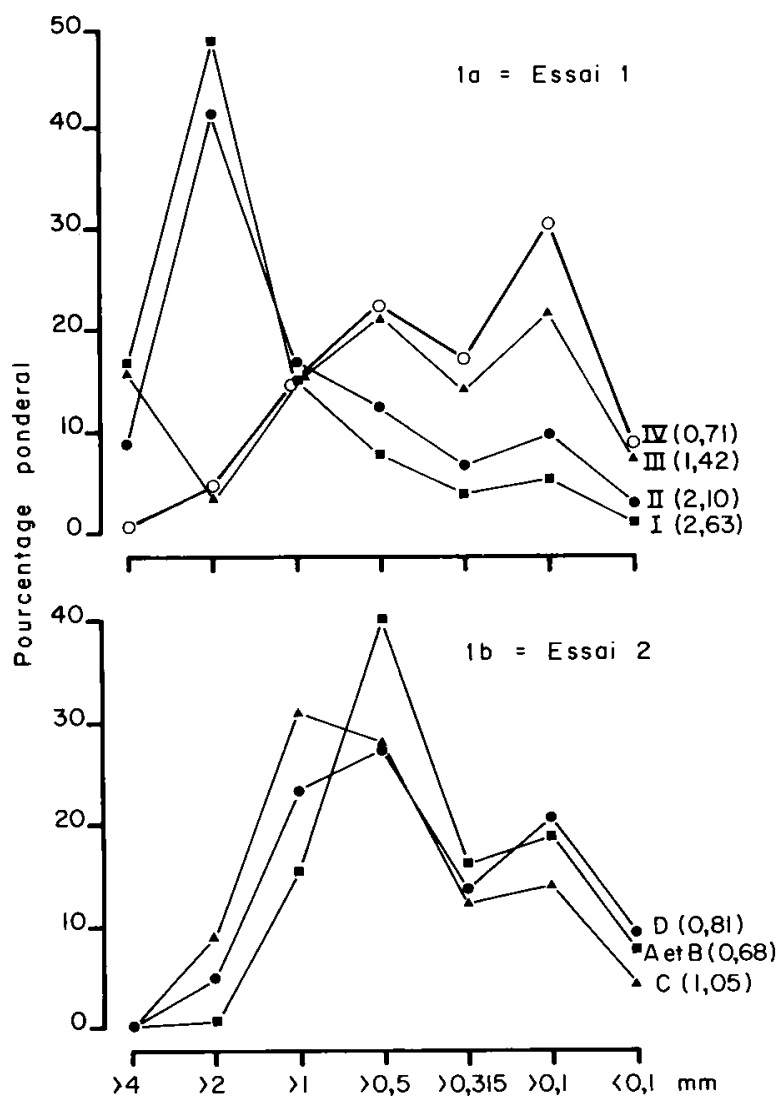

FIG. 1

Profils granulométriques des aliments expérimentaux (résultats exprimés en p. 100 pondéral de chaque fraction - ( ): granulométrie moyenne).

Granulometry of experimental diets (weight of each fraction in p. $100-():$ mean granulometry).

Dans l'essai 2, l'enregistrement du taux de mortalité par lot révèle une influence significative de la forme de présentation de l'aliment, intervenant tardivement entre 50 et 71 jours d'âge : les lots $C$ et $D$ sont les plus affectés. Il est à noter que les animaux de ces lots ingèrent des aliments dont le pourcentage de particules grossières est plus élevé que dans les deux autres lots de l'essai.

La comparaison pour des aliments de même granulométrie, présentés sous forme de granulés ou de farine ne permet pas de mettre en évidence pour nos effectifs un effet significatif sur la mortalité mais on relève une moindre atteinte pulmonaire chez les sujets ingérant la farine. 


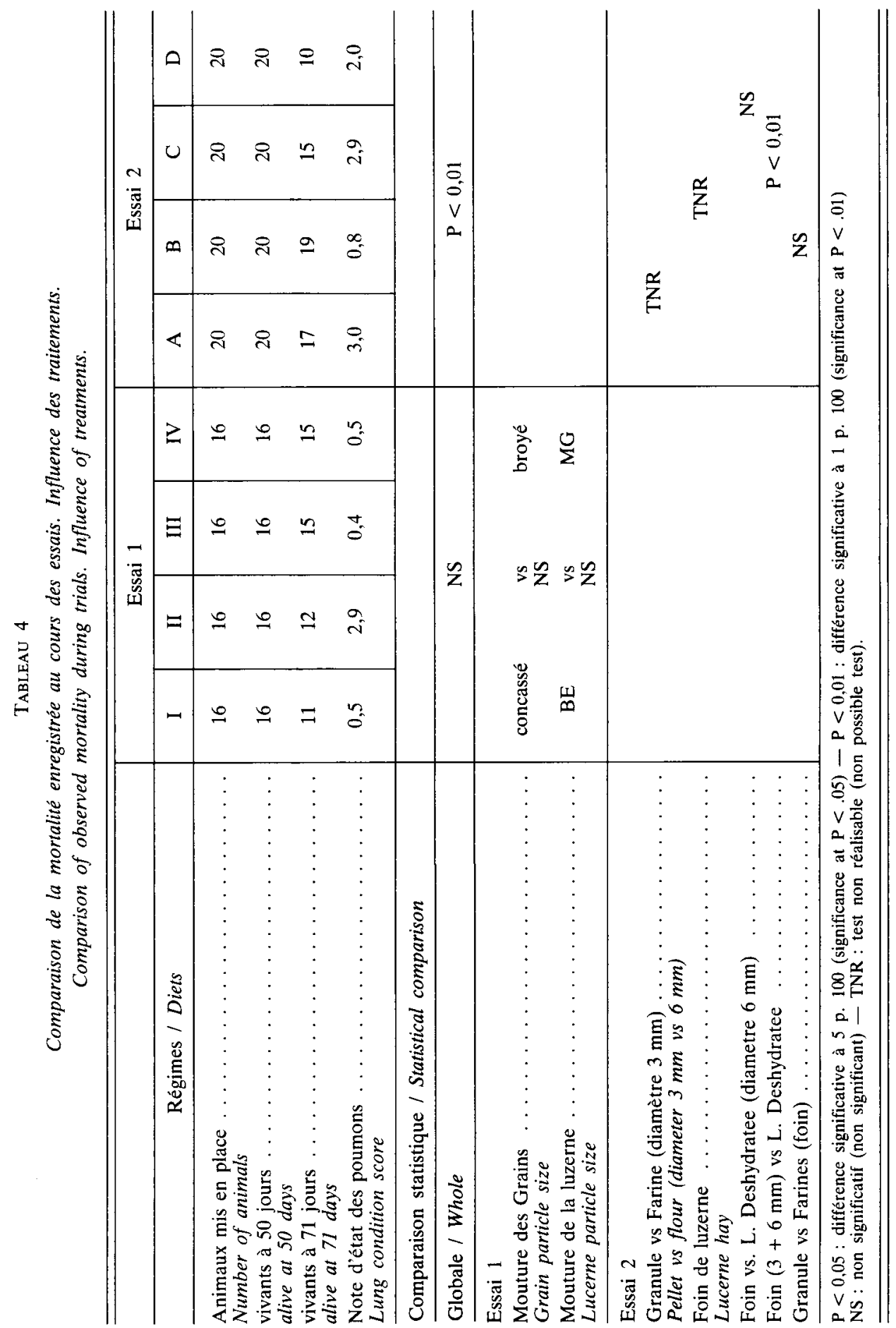


TABLEAU 5

Essai 1. Performances zootechniques: gain de poids, quantités consommées, résultats d'abattage (moyenne, écart-type de la moyenne).

Weight gain, feed intake, slaughter performance (mean, standard error of the mean).

\begin{tabular}{|c|c|c|c|c|c|c|c|c|}
\hline \multicolumn{2}{|c|}{ Régimes / Diets } & $\mathbf{I}$ & II & III & IV & \multirow{2}{*}{\multicolumn{3}{|c|}{$\begin{array}{l}\text { Ecarts moyens dus aux effets } \\
\text { Mean deviation of effects }\end{array}$}} \\
\hline \multirow{2}{*}{$\begin{array}{l}\text { Présentation } \\
\text { Physical form }\end{array}$} & \multirow{2}{*}{$\begin{array}{r}\text { des grains } \\
\text { de la } \\
\text { luzerne }\end{array}$} & \multicolumn{2}{|c|}{ concassé } & \multicolumn{2}{|c|}{ broyé } & & & \\
\hline & & Eclat & Gross. & Eclat & Gross. & $E / G$ & $\mathrm{C} / \mathrm{B}$ & Inter \\
\hline \multirow[t]{4}{*}{$\begin{array}{l}\text { Poids }(\mathrm{g}) \\
\text { Live weight }\end{array}$} & (g) $\begin{array}{r}29 \text { jours } \\
\text { days }\end{array}$ & $\begin{array}{r}555 \\
27\end{array}$ & $\begin{array}{r}554 \\
24\end{array}$ & $\begin{array}{r}564 \\
26\end{array}$ & $\begin{array}{r}551 \\
24\end{array}$ & - & - & - \\
\hline & $\begin{array}{r}36 \text { jours } \\
\text { days }\end{array}$ & $\begin{array}{r}816 \\
27\end{array}$ & $\begin{array}{r}825 \\
23\end{array}$ & $\begin{array}{r}826 \\
28\end{array}$ & $\begin{array}{r}849 \\
29\end{array}$ & NS & NS & NS \\
\hline & $\begin{array}{r}50 \text { jours } \\
\text { days }\end{array}$ & $\begin{array}{r}1273 \\
39\end{array}$ & $\begin{array}{r}1259 \\
42\end{array}$ & $\begin{array}{r}1279 \\
50\end{array}$ & $\begin{array}{r}1365 \\
34\end{array}$ & NS & NS & \multirow{2}{*}{$\begin{array}{c}\text { NS } \\
P<, 05\end{array}$} \\
\hline & $\begin{array}{r}71 \text { jours } \\
\text { days }\end{array}$ & $\begin{array}{r}1987 \\
81\end{array}$ & $\begin{array}{r}1859 \\
36\end{array}$ & $\begin{array}{r}1947 \\
68\end{array}$ & $\begin{array}{r}2032 \\
45\end{array}$ & NS & NS & \\
\hline \multirow{2}{*}{$\begin{array}{l}\text { Gain } \\
\text { moyen } \\
\text { quotidien } \\
(\mathrm{g} / \mathrm{j})\end{array}$} & $\begin{array}{r}29-36 \text { jours } \\
\text { days }\end{array}$ & $\begin{array}{r}37,2 \\
1,5\end{array}$ & $\begin{array}{r}38,8 \\
1,9\end{array}$ & $\begin{array}{r}37,4 \\
2,3\end{array}$ & $\begin{array}{r}42,5 \\
1,8\end{array}$ & \multirow{2}{*}{$\mathrm{P}<, 05$} & NS & NS \\
\hline & $\begin{array}{r}36-50 \begin{array}{r}\text { jours } \\
\text { days }\end{array} \\
\text { - }\end{array}$ & $\begin{array}{r}32,9 \\
2,3\end{array}$ & $\begin{array}{r}33,2 \\
2,4\end{array}$ & $\begin{array}{r}32,4 \\
2,0\end{array}$ & $\begin{array}{r}36,9 \\
1,5\end{array}$ & & NS & NS \\
\hline \multirow{2}{*}{$\begin{array}{l}\text { Daily mean } \\
\text { gain (g/day) }\end{array}$} & $\begin{array}{r}51-71 \text { jours } \\
\text { days }\end{array}$ & $\begin{array}{r}32,9 \\
2,3\end{array}$ & $\begin{array}{r}23,9 \\
2,4\end{array}$ & $\begin{array}{r}32,1 \\
1,0\end{array}$ & $\begin{array}{r}31,7 \\
1,5\end{array}$ & \multirow{2}{*}{$\begin{array}{c}P<, 05 \\
\text { NS }\end{array}$} & \multirow{2}{*}{$\begin{array}{c}\mathrm{P}<, 05 \\
\mathrm{NS}\end{array}$} & \multirow{2}{*}{$\begin{array}{c}\mathrm{P}<, 05 \\
\mathrm{NS}\end{array}$} \\
\hline & $\begin{array}{r}\text { 36-71 jours } \\
\text { days }\end{array}$ & $\begin{array}{r}33,1 \\
1,8\end{array}$ & $\begin{array}{r}29,0 \\
1,0\end{array}$ & $\begin{array}{r}32,1 \\
1,7\end{array}$ & $\begin{array}{r}34,0 \\
0,8\end{array}$ & & & \\
\hline \multirow{2}{*}{$\begin{array}{l}\text { Consom- } \\
\text { mation } \\
\text { alimentaire } \\
\text { (kg/animal) }\end{array}$} & $\begin{array}{r}29-36 \text { jours } \\
\text { days }\end{array}$ & $\begin{array}{r}0,71 \\
, 07\end{array}$ & $\begin{array}{r}0,54 \\
, 06\end{array}$ & $\begin{array}{r}0,69 \\
, 06\end{array}$ & $\begin{array}{r}0,69 \\
, 15\end{array}$ & NS & NS & NS \\
\hline & $\begin{array}{r}36-50 \text { jours } \\
\text { days }\end{array}$ & $\begin{array}{r}2,31 \\
, 22\end{array}$ & $\begin{array}{r}2,16 \\
, 32\end{array}$ & $\begin{array}{r}2,36 \\
, 27\end{array}$ & $\begin{array}{r}2,44 \\
, 37\end{array}$ & NS & NS & NS \\
\hline \multirow{2}{*}{$\begin{array}{l}\text { Feed intake } \\
(k g / \text { animal })\end{array}$} & $\begin{array}{r}50-71 \text { jours } \\
\text { days }\end{array}$ & $\begin{array}{r}4,67 \\
, 28\end{array}$ & $\begin{array}{r}4,46 \\
, 52\end{array}$ & $\begin{array}{r}4,29 \\
, 51\end{array}$ & $\begin{array}{r}4,12 \\
, 33\end{array}$ & NS & NS & NS \\
\hline & $\begin{array}{r}36-71 \text { jours } \\
\text { days }\end{array}$ & $\begin{array}{r}7,35 \\
, 46\end{array}$ & $\begin{array}{r}6,74 \\
, 43\end{array}$ & $\begin{array}{r}6,66 \\
, 73 \\
\end{array}$ & $\begin{array}{r}6,55 \\
, 53 \\
\end{array}$ & NS & NS & NS \\
\hline \multirow{2}{*}{$\begin{array}{l}\text { Indice } \\
\text { de consom- } \\
\text { mation }\end{array}$} & $\begin{array}{r}29-36 \text { jours } \\
\text { days }\end{array}$ & $\begin{array}{r}2,06 \\
, 21\end{array}$ & $\begin{array}{r}1,50 \\
, 09\end{array}$ & $\begin{array}{r}1,98 \\
, 15\end{array}$ & $\begin{array}{r}1,74 \\
, 23\end{array}$ & $\mathrm{P}<, 05$ & NS & NS \\
\hline & $\begin{array}{r}36-50 \text { jours } \\
\text { days }\end{array}$ & $\begin{array}{r}3,87 \\
, 17\end{array}$ & $\begin{array}{r}3,72 \\
, 18\end{array}$ & $\begin{array}{r}3,92 \\
, 22\end{array}$ & $\begin{array}{r}3,53 \\
, 28\end{array}$ & NS & NS & NS \\
\hline \multirow{2}{*}{$\begin{array}{l}\text { Feed } \\
\text { conversion } \\
\text { ratio }\end{array}$} & $\begin{array}{r}50-71 \text { jours } \\
\text { days }\end{array}$ & $\begin{array}{r}5,45 \\
, 47\end{array}$ & $\begin{array}{l}5,99 \\
1,76\end{array}$ & $\begin{array}{r}5,11 \\
, 36\end{array}$ & $\begin{array}{r}4,79 \\
, 25\end{array}$ & NS & $P<, 05$ & NS \\
\hline & $\begin{array}{r}36-71 \text { jours } \\
\text { days }\end{array}$ & $\begin{array}{r}4,81 \\
, 34\end{array}$ & $\begin{array}{r}5,08 \\
, 70\end{array}$ & $\begin{array}{r}4,64 \\
, 32\end{array}$ & $\begin{array}{r}4,29 \\
, 26\end{array}$ & NS & $\mathbf{P}<, 05$ & NS \\
\hline \multicolumn{2}{|c|}{$\begin{array}{l}\text { Rendement à l'abattage } \\
(\%) \ldots \ldots \ldots \ldots \ldots \\
\text { Dressing percentage (\%) }\end{array}$} & 61,2 & 58,5 & 60,4 & 61,7 & NS & NS & $\mathrm{P}<, 05$ \\
\hline \multicolumn{2}{|c|}{$\begin{array}{l}\text { Carcasse sans manchons } \\
\text { (g) . . . . . . . . . } \\
\text { Carcass } \\
\text { without forelegs }(g)\end{array}$} & $\begin{array}{r}1128 \\
53\end{array}$ & $\begin{array}{r}1000 \\
12\end{array}$ & $\begin{array}{r}1072 \\
56\end{array}$ & $\begin{array}{r}1145 \\
29\end{array}$ & NS & NS & $\mathrm{P}<, 05$ \\
\hline
\end{tabular}

$\mathrm{P}<, 05$ : significatif à $\mathrm{P}<0,05$ (significant at $\mathrm{P}>, 05)-$ NS : non significatif (non significant). 
TABLEAU 6

Essai 2. Performances zootechniques : gain de poids, quantités ingérées, abattage (moyenne, écart-type de la moyenne).

Trial 2. Weight gain, feed intake, slaughter performance (mean, standard error of the mean).

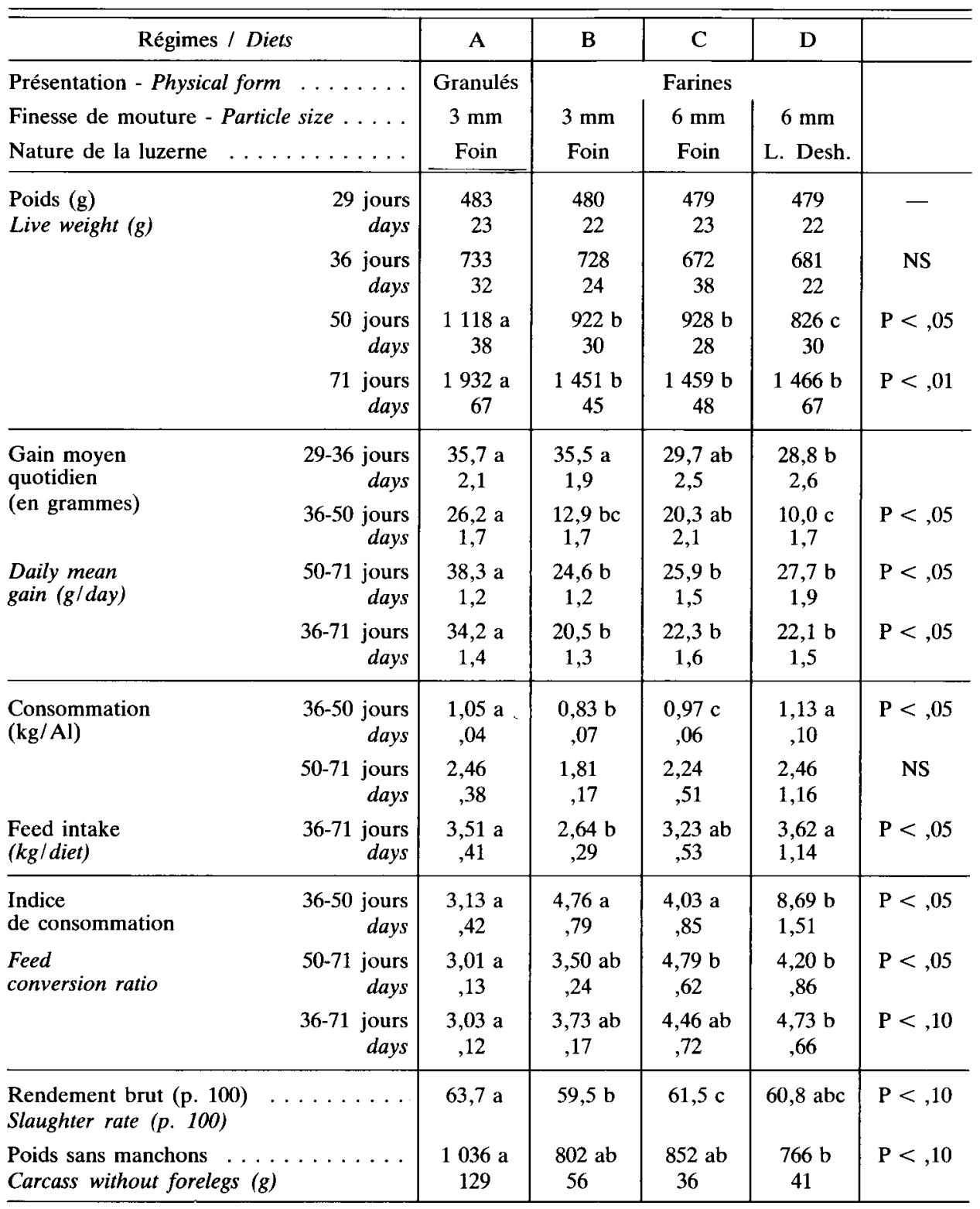

$\mathrm{P}<, 10$ : significatif à 10 p. 100 . $-\mathrm{P}<, 05$ : significatif à 5 p. $100,-\mathrm{NS}$ : non significatif

$\mathrm{a}, \mathrm{b}, \mathrm{c}:$ les données affectées d'index différents sont significativement différentes de leurs homologues $(\mathrm{P}<, 05)$.

$a, b, c$ : data with different index are significantly different from their counterparts. 
2. Quantités ingérées et gain de poids

- Essai 1 (tabl. 5)

Les animaux du lot II ont, à 71 jours, des poids vifs, des poids prêts à cuire, un rendement en carcasse significativement inférieurs $(P<0,05)$, à ceux des animaux des autres lots entre lesquels il n'y a pas de différence. Ceci correspond en particulier à un ralentissement significatif de la croissance à partir de 50 jours. Les animaux du lot IV ingérant l'aliment dont la granulométrie moyenne est la plus faible (fig. 1a) ont des gains de poids significativement supérieurs pendant la première semaine d'engraissement (29-36 jours). Les traitements n'ont aucun effet significatif sur la consommation alimentaire.

- Essai 2 (tabl. 6)

Les animaux ingérant l'aliment présenté sous forme de granulé (lot A) présentent des poids vifs et des gains moyens quotidiens très significativement supérieurs à ceux des autres lots dès l'âge de 50 jours. Ces meilleures performances de croissance sont associées à un indice de consommation inférieur.

\section{TABleau 7}

Essai 1. Caractéristiques pondérales et physico-chimiques du cacum.

Trial 1. Weight and chemical characteristics of the caecum.

\begin{tabular}{|c|c|c|c|c|c|c|}
\hline \multirow{2}{*}{$\begin{array}{l}\text { Régime / Diet } \\
\text { Grains et graines } \ldots \\
\text { Grains and seeds }\end{array}$} & I & II & III & IV & \multirow{2}{*}{\multicolumn{2}{|c|}{$\begin{array}{c}\text { Ecarts moyens } \\
\text { dus aux effets } \\
\text { Mean deviation of effects }\end{array}$}} \\
\hline & \multicolumn{2}{|c|}{$\begin{array}{l}\text { concassés } \\
\text { crushed }\end{array}$} & \multicolumn{2}{|c|}{$\begin{array}{l}\text { broyés } \\
\text { ground }\end{array}$} & & \\
\hline $\begin{array}{l}\text { Luzerne en bouchon } \\
\text { Lucerne pellets }\end{array}$ & Eclat & Gross. & Eclat & Gross. & $\mathrm{E}$ vs $\mathrm{G}$ & G. Broye \\
\hline $\begin{array}{l}\text { Poids du cacum }(\mathrm{g}) \\
\text { Caecal weight }(\mathrm{g})\end{array}$ & $\begin{array}{c}30,7 \mathrm{a} \\
, 9\end{array}$ & $\begin{array}{c}32,6 \mathrm{a} \\
1,2\end{array}$ & $\begin{array}{c}33,8 \mathrm{a} \\
1,2\end{array}$ & $\begin{array}{c}28,0 \mathrm{~b} \\
1,3\end{array}$ & $\begin{array}{l}+\quad 2,0 \\
P<, 10\end{array}$ & NS \\
\hline $\begin{array}{l}\text { Poids cæcal/PM .... } \\
\text { Caecal weight/MW } \\
(\mathrm{g} / \mathrm{kg})\end{array}$ & $\begin{array}{c}18,4 \mathrm{ab} \\
, 8\end{array}$ & $\begin{array}{c}20,4 \text { a } \\
, 8\end{array}$ & $\begin{array}{c}20,7 \text { a } \\
, 6\end{array}$ & $\begin{array}{c}16,7 \mathrm{~b} \\
, 9\end{array}$ & NS & NS \\
\hline $\begin{array}{l}\text { Contenu cæcal }(\mathrm{g}) \\
\text { Caecal content }(\mathrm{g})\end{array}$ & $\begin{array}{c}111,7 \text { a } \\
9,2\end{array}$ & $\begin{array}{c}133,4 \mathrm{~b} \\
9,8\end{array}$ & $\begin{array}{c}108,8 \text { a } \\
3,2\end{array}$ & $\begin{array}{c}114,1 \text { a } \\
6,5\end{array}$ & $\begin{array}{l}-13,6 \\
P<, 10\end{array}$ & NS \\
\hline $\begin{array}{l}\text { Matière sèche }(\%) \\
\text { Dry matter }(\%)\end{array}$ & $\begin{array}{r}23,19 \\
, 96\end{array}$ & $\begin{array}{r}22,96 \\
, 64\end{array}$ & $\begin{array}{r}21,35 \\
1,11\end{array}$ & $\begin{array}{r}23,45 \\
, 35\end{array}$ & NS & NS \\
\hline $\begin{array}{l}\text { Matière sèche/PM } \\
\text { Dry matter } / M W \\
(\mathrm{~g} / \mathrm{kg})\end{array}$ & $\begin{array}{c}14,19 \mathrm{a} \\
, 62\end{array}$ & $\begin{array}{c}18,07 \mathrm{~b} \\
1,40\end{array}$ & $\begin{array}{c}15,16 \text { a } \\
, 82\end{array}$ & $\begin{array}{c}15,83 \mathrm{a} \\
\quad, 89\end{array}$ & $\begin{array}{l}-\quad 2,28 \\
P<, 05\end{array}$ & NS \\
\hline pH & $\begin{array}{c}5,76 \text { a } \\
, 08\end{array}$ & $\begin{array}{c}6,16 \mathrm{~b} \\
, 08\end{array}$ & $\begin{array}{c}5,93 \text { a } \\
, 08\end{array}$ & $\begin{array}{c}5,86 \text { a } \\
, 05\end{array}$ & $\begin{array}{c}-0,16 \\
P<, 05\end{array}$ & NS \\
\hline
\end{tabular}

$\mathrm{P}<, 10$ : significatif à $10 \mathrm{p} .100$. - $\mathrm{P}<0,05$ : significatif à 5 p. 100 . - NS : non significatif.

$a, b, c:$ les données affectées d'index différents sont significativement différentes de leurs homologues $(\mathrm{P}<, 05)$. PM $(M W)$ : poids métabolique (metabolic weight) $=$ poids vif 0,75 .

(Cf. tabl. 6). 
En comparaison avec les animaux du lot C, ceux du lot B recevant un aliment dont la granulométrie est plus faible $(3 \mathrm{~mm}$ vs $6 \mathrm{~mm}$, fig. 1b), présentent un gain moyen quotidien significativement plus élevé pendant la première semaine d'engraissement (29-36 jours), malgré des niveaux d'ingestion inférieurs, et à l'abattage, un rendement en carcasse significativement réduit.

La forte mortalité enregistrée pour les animaux du lot $\mathrm{D}$ ingérant un aliment dont la granulométrie est hétérogène et dont la valeur alimentaire est plus faible ne permet pas d'interpréter les résultats de croissance et d'abattage.

\section{Prélèvement du cacum après abattage}

Sur l'ensemble des résultats (tabl. 7 et 8), deux lots d'animaux (lots II et D) se distinguent nettement: le poids de leur contenu cacal est supérieur, le pH de ce contenu est plus élevé (supérieur à 6). Les animaux de ces lots ont présenté les performances zootechniques les plus faibles. Dans le lot $\mathrm{D}$ en particulier, la mortalité est très élevée, la réplétion du cæcum est importante, les fermentations sont caractérisées par un faible pourcentage molaire d'acide butyrique.

Dans l'essai 2 (tabl. 8), l'ingestion de l'aliment sous forme de granulé est associée à un contenu cæcal sec très significativement réduit et plus alcalin, en comparaison avec le même aliment présenté sous forme de farine. Les taux d'acides gras volatils totaux du contenu cacal (essai 2) ne sont influencés ni par la forme de présentation (lot A vs lot B) ni par la nature de la luzerne (lot $\mathrm{C}$ vs lot D) mais se différencient significativement en fonction de la finesse de mouture (concentrations plus faibles avec les grosses moutures).

\section{Discussion et Conclusion}

\section{A. Troubles pathologiques}

Les effectifs des animaux mis en expérience sont insuffisants pour conclure statistiquement à un effet spécifique de la forme de présentation ou de granulométrie des aliments sur la viabilité des animaux.

Seule la mortalité élevée enregistrée chez les animaux du lot D recevant un aliment à base de luzerne déshydratée à forte teneur en fibres (NDF 46,3 p. $100 \mathrm{MS}$ ) et à faible taux azoté (14,3 p. $100 \mathrm{MS}$ ) paraît significative. En accord avec les observations de Morisse (1982), le pH du contenu cacal augmente alors que le pourcentage molaire en acide butyrique des acides gras volatils totaux de ce contenu diminue. Par ailleurs, l'ingestion d'un tel aliment entraîne une augmentation significative du poids du contenu cæcal traduisant probablement une stase à ce niveau et une vidange ralentie de l'organe associée à des modifications de la motricité (PAIRet et al., 1986).

\section{B. Influence de la forme de présentation}

En comparaison avec une présentation sous forme de farine, la granulation de l'aliment améliore nettement les performances zootechniques : gain moyen quotidien de $34 \mathrm{~g} / \mathrm{j}$ par rapport à $21 \mathrm{~g} / \mathrm{j}$ (période $36-71 \mathrm{j}$ ), rendement carcasse 63,7 p. 100 par rapport à 59,5 p. 100 , indice de consommation 3,03 par rapport à 3,73 (période 36-71 j). Ceci confirme les observations de Lebas (1973) et de KING (1974). 


\section{TABLEAU 8}

Essai 2. Caractéristiques pondérales et physico-chimiques du cacum.

Trial 2. Weight and chemical characteristics of the caecum.

\begin{tabular}{|c|c|c|c|c|c|}
\hline Régime / Diet & A & B & $\mathrm{C}$ & D & \\
\hline 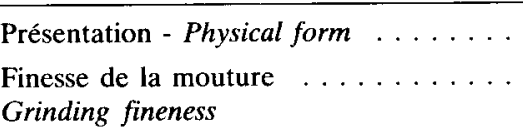 & $\begin{array}{c}\text { Granulés } \\
3 \mathrm{~mm}\end{array}$ & $3 \mathrm{~mm}$ & $\begin{array}{c}\text { Farines } \\
6 \mathrm{~mm}\end{array}$ & $6 \mathrm{~mm}$ & \\
\hline Nature de la luzerne - Type of lucerne . & Foin & Foin & Foin & L. Desh. & \\
\hline $\begin{array}{l}\text { Poids du cæcum }(\mathrm{g}) \\
\text { Caecal weight }(g)\end{array}$ & $\begin{array}{r}34,9 \text { a } \\
1,7\end{array}$ & $\begin{aligned} 29,0 & \text { bc } \\
1,5 & \end{aligned}$ & $\begin{array}{r}25,6 \mathrm{c} \\
1,0\end{array}$ & $\begin{array}{r}31,3 \text { ab } \\
1,3\end{array}$ & $\mathrm{P}<, 01$ \\
\hline $\begin{array}{l}\text { Poids cacal/poids met. } \ldots \ldots \\
\text { Caecal weight/Metabolic W. }(\mathrm{g} / \mathrm{kg})\end{array}$ & $\begin{array}{r}21,5 \text { a } \\
1,0\end{array}$ & $\begin{array}{rr}21,5 & \text { a } \\
0,9 & \end{array}$ & $\begin{array}{cc}18,6 \\
0,7\end{array}$ & $\begin{array}{c}23,8 \\
0,7\end{array}$ & $\mathbf{P}<, 05$ \\
\hline $\begin{array}{l}\text { Contenu cxcal }(\mathrm{g}) \text {. } \\
\text { Caecal contents }(\mathrm{g})\end{array}$ & $\begin{array}{r}87,9 \text { a } \\
6,6\end{array}$ & $\begin{aligned} 99,3 & \text { a } \\
2,8 & \end{aligned}$ & $\begin{array}{r}91,6 \text { a } \\
6,0\end{array}$ & $\begin{array}{r}127,4 \text { b } \\
11,5\end{array}$ & $\mathrm{P}<, 01$ \\
\hline $\begin{array}{l}\text { Matière sèche }(\%) \\
\text { Dry matter }(\%)\end{array}$ & $\begin{array}{r}21,3 \\
0,4\end{array}$ & $\begin{array}{r}21,0 \\
0,4\end{array}$ & $\begin{array}{r}21,5 \\
0,5\end{array}$ & $\begin{array}{r}21,2 \\
0,4\end{array}$ & NS \\
\hline $\begin{array}{l}\text { Matière sèche/Poids met. } \ldots \\
\text { Dry matter/Metabolic } W .(\mathrm{g} / \mathrm{kg})\end{array}$ & $\begin{array}{r}10,4 \text { a } \\
0,4\end{array}$ & $\begin{array}{rl}15,6 & b \\
1,0 & \end{array}$ & $\begin{array}{r}14,5 \mathrm{~b} \\
, 8\end{array}$ & $\begin{array}{r}20,7 \mathrm{c} \\
1,4\end{array}$ & $\mathbf{P}<, 01$ \\
\hline $\mathrm{pH}$ & $\begin{array}{l}5,95 \text { a } \\
0,07\end{array}$ & $\begin{array}{l}5,66 \text { b } \\
0,09\end{array}$ & $\begin{array}{l}5,56 \mathrm{~b} \\
0,09\end{array}$ & $\begin{array}{l}6,37 \mathrm{c} \\
0,08\end{array}$ & $\mathrm{P}<, 01$ \\
\hline $\begin{array}{l}\text { Acides gras volatils totaux } \ldots \ldots \ldots \ldots \\
\text { Total volatile fatty acids } \\
\text { (micro } \mathrm{M} / \mathrm{g} \text { matière sèche) }\end{array}$ & $\begin{array}{rr}330 & \mathbf{a} \\
27 & \end{array}$ & $\begin{array}{r}351 \\
25\end{array}$ & $\begin{array}{rr}271 & b \\
29 & \end{array}$ & $\begin{array}{rr}262 & b \\
19 & \end{array}$ & $\mathbf{P}<, 05$ \\
\hline Acide acétique $(\mathrm{C} 2)$ & $\begin{array}{r}208 \\
21\end{array}$ & $\begin{array}{r}224 \\
17\end{array}$ & $\begin{array}{l}176 \\
21\end{array}$ & $\begin{array}{c}179 \\
16\end{array}$ & NS \\
\hline Acide Propionique $(\mathrm{C} 3) \ldots \ldots \ldots$ & $\begin{array}{r}31 \\
5\end{array}$ & $\begin{array}{r}28 \\
4\end{array}$ & $\begin{array}{r}22 \\
3\end{array}$ & $\begin{array}{r}28 \\
5\end{array}$ & NS \\
\hline Acide Butyrique (C4). & $\begin{array}{rr}91 & \mathrm{a} \\
9 & \end{array}$ & $\begin{array}{r}100 \quad \mathrm{a} \\
9\end{array}$ & $\begin{array}{rr}73 & b \\
8 & \end{array}$ & $\begin{array}{ll}47 & c \\
11 & \end{array}$ & $\mathbf{P}<, 01$ \\
\hline $\begin{array}{lll}\begin{array}{l}\text { Pourcentage molaire } \\
\text { Molar percentage }\end{array} & - \text { (C2) } & \ldots \ldots \\
& - \text { (C3) } & \ldots \ldots \\
& & \text { (C4) } \ldots \ldots\end{array}$ & $\begin{array}{rr}62,4 & \text { a } \\
9,3 & \\
28,3 & \text { a }\end{array}$ & $\begin{array}{rr}63,8 & \text { a } \\
7,7 & \\
28,4 & \text { a }\end{array}$ & $\begin{array}{rr}64,5 & \text { a } \\
8,2 & \\
27,3 & \text { a }\end{array}$ & $\begin{array}{l}70,6 \quad b \\
11,5 \\
17,8 \quad b\end{array}$ & $\begin{array}{l}\mathrm{P}<, 05 \\
\quad \mathrm{NS} \\
\mathrm{P}<, 05\end{array}$ \\
\hline
\end{tabular}

$\mathrm{P}<, 05$ : significatif à 5 p. $100 .-\mathrm{P}<, 01$ : significatif à 1 p. 100 . - NS : non significatif.

$\mathrm{a}, \mathrm{b}, \mathrm{c}:$ les données affectées d'index différents sont significativement différentes de leurs homologues $(\mathrm{P}<, 05)$. (Cf. tabl. 6).

La distribution aux animaux d'un aliment farine homogène entraîne une diminution importante des quantités ingérées, ce qu'avaient déjà mentionné Dehalle et Lebas (1981), attribuable certainement aux difficultés d'écoulement de la farine dans des trémies prévues pour une alimentation granulée. Cette ingestion insuffisante d'éléments nutritifs entraîne une sous-nutrition énergétique et azotée expliquant la détérioration de l'indice de consommation, en accord avec les conclusions de Machin et al. (1980). Par contre, nos résultats infirment les idées reçues sur une relation de cause à effet entre l'ingestion de farine et l'apparition de troubles pulmonaires. Le cæcum des animaux 
ingérant l'aliment granulé, dans nos conditions d'abattage (10 heures du matin), est moins rempli que celui des animaux ingérant un aliment de même finesse de mouture mais présenté sous forme de farine. La forme de présentation a donc, indépendamment de la granulométrie, un effet propre sur l'état de réplétion de cet organe en relation avec ses caractéristiques physiques et le rythme d'ingestion lié au comportement alimentaire (LeBAS et LAPLACE, 1977).

\title{
C. Influence de la finesse de mouture
}

Les aliments I, II et III de l'essai 1 sont relativement grossiers (taille moyenne des particules supérieure à $1,4 \mathrm{~mm}$ ) et hétérogènes dans la mesure où les différentes matières premières les composant restent aisément identifiables. La distribution de tels aliments permet un tri des matières premières par les animaux d'où une détérioration de l'indice de consommation sans incidence pathologique majeure dans cet essai.

Dans les deux essais, en accord avec LAPLACE et LeBAs (1977), les granulométries les plus fines (taille moyenne des particules de $0,70 \mathrm{~mm}$; lots IV vs lot III dans l'essai 1 ; lot $\mathrm{B}$ vs lot $\mathrm{C}$ dans l'essai 2) sont associées à un meilleur démarrage de la croissance (26-36 j), à des indices de consommation plus faibles, à des taux d'acides gras volatils des contenus cæcaux plus élevés en particulier celui de l'acide butyrique. Cet accroissement de la production d'acides gras volatils peut être relié à une fermentescibilité accrue des fragments alimentaires rendus plus accessibles à la micropopulation cæcale par la réduction de leur taille. Le facteur limitant semble donc être la capacité d'ingestion de matière sèche comme l'ont déjà souligné Machin et al. (1980).

En conclusion, la farine ne semble pas provoquer de problèmes pathologiques spécifiques par rapport aux aliments granulés. La sous-consommation observée par rapport au lot nourri en granulés semblerait indiquer que le lapin n'ajuste plus sa consommation sur l'énergie quand il est nourri en farine, mais sur des quantités ou des volumes comme les herbivores (Machin et al., 1980).

Des particules fines $(0,70 \mathrm{~mm}$ en moyenne) favorisent une meilleure efficacité alimentaire, d'une part en limitant le comportement de tri, d'autre part en favorisant la dégradation des fragments alimentaires dans le tractus digestif.

Reçu en avril 1986.

Accepté en septembre 1986.

\begin{abstract}
Summary
Effect of the physical form and grinding fineness of feeds upon growth and caecal function in growing rabbits
\end{abstract}

The effect of the physical form of feeds (pellets vs flours) (tabl. 1) and of their grinding fineness (fig. 1) prior to pelleting upon the growth performance and caecal characteristics of growing rabbits, were investigated in two trials.

Flours induced an underconsumption of feed, but no respiratory disorders (tabl. 4).

The heterogeneity of unpelleted mixed diets allowed a sorting of feedstuffs and a reduction of the feed conversion ratio (tabl. 5). Pellets prepared with finely ground feeds gave the best 
performance. At slaughter, examination of the caecal contents of animals fed these pellets showed a lower caecal $\mathrm{pH}$ and a higher volatile fatty acid (VFA) concentration (tabl. 8).

Conversely, the lowest performance associated with a high caecal $\mathrm{pH}$ and a fermentary deviation of VFA proportions (reduced C4 p. 100, increased $C 2$ p. 100) were observed in animals receiving coarse flours.

Key words : Nutrition, rabbit, flour, granulometry, caecum.

\section{Références bibliographiques}

Aumaitre A., Melcion J.P., Vaissade P., Peiniau J., 1977. L'agglomération des aliments de sevrage précoce du porcelet : conséquences nutritionnelles de la technologie de préparation. $J$. Rech. Porcine en France, 9, 211-215, INRA-I.T.P. Editeurs.

BaIRD D.M., 1973. Influence of pelleting swine diets on metabolizable energy, growth and carcass characteristics. J. Anim. Sci., 36, 516-521.

Braude R., Rowell J.G., 1966. Comparison of meal and pellets for growing pigs fed either in throughs or off the floor. J. Agr. Sci., 67, 53-68.

Communauté européEnne, 1971. a) Méthodes de dosage officielles dans la Communauté européenne : dosage des cendres brutes. Journal officiel Communautés européennes, L. 155 du 12 07-1971.

Communauté européenne, 1971. b) Méthodes de dosage officielles dans la Communauté européenne : dosage de l'humidité, des matières grasses brutes. Journal officiel des Communautés européennes, L. 279 du 20-12-1972.

Communauté européenne, 1972. Méthodes de dosage officielles dans la Communauté européenne : dosage des protéines brutes. Journal officiel des Communautés européennes, L. 123 du 29-05-1972.

Communauté européenne, 1973. Méthodes de dosage officielles dans la Communauté européenne : dosage de la cellulose brute. Journal officiel des Communautés européennes, L. 83 du 30-03-1973.

Dehalle C., Lebas $F_{\text {., }}$ 1981. Incidences de régimes alimentaires à base de luzerne sur la composition en acides gras volatils du contenu digestif chez le lapin. Reprod. Nutr. Dévelop., 21, 867.

I.T.P., 1981. Notations des lésions d'atélectasie pulmonaire chez le porc. Document I.T.P., réf. IV-018.

King J.O.L., 1974. The effects of pelleting rations with and without an antibiotic on the growth rate of rabbits. Vet. Rec., 94, 586-588.

Laplace J.P., Lebas F., 1977. Le transit digestif chez le lapin. VII. Influence de la finesse du broyage des constituants d'un aliment granulé. Ann. Zootech., 26, 413-420.

Lebas F., 1973. Possibilité d'alimentation du lapin en croissance avec des régimes présentés sous forme de farine. Ann. Zootech., 22, 249-257.

Lebas F., Laplace J.P., 1977. Le transit digestif chez le lapin. Influence de la granulation des aliments. Ann. Zootech., 26, 83-91.

Machin M., Butcher C., Owen E., Bryant M., Owen M.E., 1980. The effects of dietary metabolizable energy concentration and physical form of the diet on the performance of growing rabbits. $2^{\mathrm{e}}$ Congr. Int. Cunicole Barcelone, vol. II, 65-75.

Morin, Seroux M., Piganeau P., 1979. Méthodes d'expérimentation sur le lapin. Perspectives Agricoles, $\mathrm{n}^{\circ}$ 23, 64-72.

Morisse J.P., 1982. L'alimentation du lapin : composante primordiale de l'équilibre digestif. Rôle des glucides. Rev. Alim. Anim., $n^{\circ}$ 354, 21-24.

Pairet M., Bouyssou Th., Auvergne A., Candau M., Ruckebusch Y., 1986. Stimulation physico-chimique d'origine alimentaire et motricité digestive chez le lapin. Reprod. Nutr. Develop., 26 (1A), 85-96.

VAN Soest D.J., 1967. Development of a comprehensive system of feeds analyses and its applications of forage. J. Anim. Sci., 26, 119-128. 